\title{
$f$-asymptotically lacunary ideal equivalence of double sequences
}

Nimet Pancaroğlu Akın ${ }^{* *}$

\author{
"Correspondence: \\ npancaroglu@aku.edu.tr \\ ${ }^{1}$ Deparment of Mathematics and \\ Science Education, Faculty of \\ Education, Afyon Kocatepe \\ University, Afyonkarahisar, Turkey
}

\begin{abstract}
In this study, we present the notions of $f$-asymptotically $\mathcal{I}_{2}$-equivalence, strongly $f$-asymptotically $\mathcal{I}_{2}$-equivalence, $f$-asymptotically lacunary $\mathcal{I}_{2}$-equivalence, and strongly $f$-asymptotically lacunary $\mathcal{I}_{2}$-equivalence of double sequences and investigate some relationships between them. Also, we examine some relationships between strongly $f$-asymptotically $\mathcal{I}_{2}$-equivalence and asymptotically $\mathcal{I}_{2}$-statistical equivalence and between strongly $f$-asymptotically lacunary $\mathcal{I}_{2}$-equivalence and asymptotically lacunary $\mathcal{I}_{2}$-statistical equivalence of double sequences.
\end{abstract}

MSC: 40A05; 40A35

Keywords: Asymptotically equivalence; Lacunary sequence; $\mathcal{I}_{2}$-equivalence; Double sequence; Modulus function

\section{Introduction and background}

Throughout the paper, $\mathbb{N}$ is the set of all positive integers, and $\mathbb{R}$ is the set of all real numbers. The concept of convergence of a sequence of real numbers has been extended to statistical convergence independently by Fast [14] and Schoenberg [39]. Fridy and Orhan [15] studied lacunary statistical convergence. This concept was extended to the double sequences by Mursaleen and Edely [28]. A lot of development have been made in this area after the works of $[1,2,13,25-27]$.

The idea of $\mathcal{I}$-convergence was introduced by Kostyrko, Šalát, and Wilczyński [20] as a generalization of statistical convergence. Das et al. [4] introduced the concept of $\mathcal{I}$ convergence of double sequences in a metric space and studied some properties of this convergence. A lot of development have been made in this area after the works of [5-11, 29-32, 40, 44].

Marouf [24] presented definitions for asymptotically equivalent and asymptotic regular matrices. Patterson [35] presented asymptotically statistically equivalent sequences for nonnegative summability matrices. Dündar et al. [12] defined asymptotically $\mathcal{I}_{2}^{\sigma}$ equivalent, asymptotically invariant equivalent, strongly asymptotically invariant equivalent, and $p$-strongly asymptotically invariant equivalent for double sequences. Ulusu and Dündar [42] introduced the concepts of asymptotically lacunary $\mathcal{I}_{2}$-invariant equivalence, asymptotically lacunary $\sigma_{2}$-equivalence, and asymptotically lacunary invariant $S_{2}$ equivalence of double sequences. Hazarika and Kumar [16] studied asymptotically double lacunary statistically equivalent sequences in ideal context.

(c) The Author(s) 2019. This article is distributed under the terms of the Creative Commons Attribution 4.0 International License (http://creativecommons.org/licenses/by/4.0/), which permits unrestricted use, distribution, and reproduction in any medium, provided you give appropriate credit to the original author(s) and the source, provide a link to the Creative Commons license, and indicate if changes were made. 
The modulus function was introduced by Nakano [33]. Maddox [23], Pehlivan [37], and many authors used the modulus function $f$ to define some new concepts and inclusion theorems. Kumar and Sharma [21] studied lacunary equivalent sequences by ideals and the modulus function. Also, several authors define some new concepts and give inclusion theorems using a modulus function $f$ (see $[17,18]$ ).

Now we recall the basic concepts and some definitions (see $[3,15,19,20,22-24,34-38$, $41,43])$.

By a lacunary sequence we mean an increasing integer sequence $\theta=\left\{k_{r}\right\}$ such that $k_{0}=0$ and $h_{r}=k_{r}-k_{r-1} \rightarrow \infty$ as $r \rightarrow \infty$. Throughout the paper, we let $\theta=\left\{k_{r}\right\}$ be a lacunary sequence.

A double sequence $\theta_{2}=\left\{\left(k_{r}, j_{u}\right)\right\}$ is called a double lacunary sequence if there exist two increasing sequences of integers such that $k_{0}=0, h_{r}=k_{r}-k_{r-1} \rightarrow \infty$ and $j_{0}=0, \bar{h}_{u}=$ $j_{u}-j_{u-1} \rightarrow \infty$ as $r, u \rightarrow \infty$. We further use the following notations:

$$
\begin{aligned}
& k_{r u}=k_{r} j_{u}, \quad h_{r u}=h_{r} \bar{h}_{u}, \quad I_{r u}=\left\{(k, j): k_{r-1}<k \leq k_{r} \text { and } j_{u-1}<j \leq j_{u}\right\}, \\
& q_{r}=\frac{k_{r}}{k_{r-1}} \text { and } q_{u}=\frac{j_{u}}{j_{u-1}} .
\end{aligned}
$$

Throughout the paper, we let $\theta_{2}=\left\{\left(k_{r}, j_{u}\right)\right\}$ be a double lacunary sequence.

A family of sets $\mathcal{I} \subseteq 2^{\mathbb{N}}$ is called an ideal if and only if

(i) $\emptyset \in \mathcal{I}$,

(ii) for all $A, B \in \mathcal{I}$, we have $A \cup B \in \mathcal{I}$,

(iii) for each $A \in \mathcal{I}$ and each $B \subseteq A$, we have $B \in \mathcal{I}$.

An ideal is called nontrivial if $\mathbb{N} \notin \mathcal{I}$, and a nontrivial ideal is called admissible if $\{n\} \in \mathcal{I}$ for each $n \in \mathbb{N}$. Throughout the paper, we let $\mathcal{I}$ be an admissible ideal.

A nontrivial ideal $\mathcal{I}_{2}$ of $\mathbb{N} \times \mathbb{N}$ is called a strongly admissible ideal if $\{i\} \times \mathbb{N}$ and $\mathbb{N} \times\{i\}$ belong to $\mathcal{I}_{2}$ for each $i \in N$.

Throughout the paper, we let $\mathcal{I}_{2}$ be a strongly admissible ideal in $\mathbb{N} \times \mathbb{N}$.

It is evident that a strongly admissible ideal is admissible.

We denote $\mathcal{I}_{2}^{0}=\{A \subset \mathbb{N} \times \mathbb{N}:(\exists m(A) \in \mathbb{N})(i, j \geq m(A) \Rightarrow(i, j) \notin A)\}$. Then $\mathcal{I}_{2}^{0}$ is a strongly admissible ideal, and clearly an ideal $\mathcal{I}_{2}$ is strongly admissible if and only if $\mathcal{I}_{2}^{0} \subset \mathcal{I}_{2}$.

Two nonnegative sequences $x=\left(x_{k}\right)$ and $y=\left(y_{k}\right)$ are said to be asymptotically equivalent if $\lim _{k \rightarrow \infty} \frac{x_{k}}{y_{k}}=1$ (denoted by $x \sim y$ ).

Two nonnegative sequences $x=\left(x_{k}\right)$ and $y=\left(y_{k}\right)$ are said to be asymptotically statistically equivalent of multiple $L$ if for every $\varepsilon>0$,

$$
\lim _{n \rightarrow \infty} \frac{1}{n}\left|\left\{k \leq n:\left|\frac{x_{k}}{y_{k}}-L\right| \geq \varepsilon\right\}\right|=0
$$

(denoted by $x \stackrel{S_{L}}{\sim} y$ ) and simply asymptotically statistically equivalent, if $L=1$.

Two nonnegative sequences $x=\left(x_{k}\right)$ and $y=\left(y_{k}\right)$ are said to be strongly asymptotically equivalent of multiple $L$ with respect to the ideal $\mathcal{I}$ if for every $\varepsilon>0$,

$$
\left\{n \in \mathbb{N}: \frac{1}{n} \sum_{k=1}^{n}\left|\frac{x_{k}}{y_{k}}-L\right| \geq \varepsilon\right\} \in \mathcal{I}
$$


(denoted by $x_{k} \stackrel{\mathcal{I}(\omega)}{\sim} y_{k}$ ) and simply strongly asymptotically equivalent with respect to the ideal $\mathcal{I}$ if $L=1$.

Two nonnegative sequences $x=\left(x_{k}\right)$ and $y=\left(y_{k}\right)$ are said to be strongly asymptotically lacunary equivalent of multiple $L$ with respect to the ideal $\mathcal{I}$ if for every $\varepsilon>0$,

$$
\left\{r \in \mathbb{N}: \frac{1}{h_{r}} \sum_{k \in I_{r}}\left|\frac{x_{k}}{y_{k}}-L\right| \geq \varepsilon\right\} \in \mathcal{I}
$$

(denoted by $x_{k} \stackrel{\mathcal{I}\left(N_{\theta}\right)}{\sim} y_{k}$ ) and simply strongly asymptotically lacunary $\mathcal{I}$-equivalent with respect to the ideal $\mathcal{I}$ if $L=1$.

Two nonnegative sequences $x=\left(x_{k}\right)$ and $y=\left(y_{k}\right)$ are said to be asymptotically lacunary statistically equivalent of multiple $L$ with respect to the ideal $\mathcal{I}$ if for all $\varepsilon>0$ and $\gamma>0$,

$$
\left\{r \in \mathbb{N}: \frac{1}{h_{r}}\left|\left\{k \in I_{r}:\left|\frac{x_{k}}{y_{k}}-L\right| \geq \varepsilon\right\}\right| \geq \gamma\right\} \in \mathcal{I}
$$

(denoted by $x_{k} \stackrel{\mathcal{I}\left(\mathcal{S}_{\theta}\right)}{\sim} y_{k}$ ) and simply asymptotically lacunary $\mathcal{I}$-statistically equivalent if $L=1$.

A function $f:[0, \infty) \rightarrow[0, \infty)$ is called a modulus if

1. $f(x)=0$ if and if only if $x=0$,

2. $f(x+y) \leq f(x)+f(y)$,

3. $f$ is increasing, and

4. $f$ is continuous from the right at 0 .

A modulus may be unbounded (for example, $f(x)=x^{p}, 0<p<1$ ) or bounded (for example, $\left.f(x)=\frac{x}{x+1}\right)$.

Let $f$ be a modulus function. Two nonnegative sequences $x=\left(x_{k}\right)$ and $y=\left(y_{k}\right)$ are said to be $f$-asymptotically equivalent of multiple $L$ with respect to the ideal $\mathcal{I}$ if for every $\varepsilon>0$,

$$
\left\{k \in \mathbb{N}: f\left(\left|\frac{x_{k}}{y_{k}}-L\right|\right) \geq \varepsilon\right\} \in \mathcal{I}
$$

(denoted by $x_{k} \stackrel{\mathcal{I}(f)}{\sim} y_{k}$ ) and simply $f$-asymptotically $\mathcal{I}$-equivalent if $L=1$.

Let $f$ be a modulus function. Two nonnegative sequences $x=\left(x_{k}\right)$ and $y=\left(y_{k}\right)$ are said to be strongly $f$-asymptotically equivalent of multiple $L$ with respect to the ideal $\mathcal{I}$ if for every $\varepsilon>0$,

$$
\left\{n \in \mathbb{N}: \frac{1}{n} \sum_{k=1}^{n} f\left(\left|\frac{x_{k}}{y_{k}}-L\right|\right) \geq \varepsilon\right\} \in \mathcal{I}
$$

(denoted by $\left.x_{k} \stackrel{\mathcal{I}\left(\omega_{f}\right)}{\sim} y_{k}\right)$ ) and simply strongly $f$-asymptotically $\mathcal{I}$-equivalent if $L=1$.

Let $f$ be a modulus function. Two nonnegative sequences $x=\left(x_{k}\right)$ and $y=\left(y_{k}\right)$ are said to be strongly $f$-asymptotically lacunary equivalent of multiple $L$ with respect to the ideal $\mathcal{I}$ if for every $\varepsilon>0$,

$$
\left\{r \in \mathbb{N}: \frac{1}{h_{r}} \sum_{k \in I_{r}} f\left(\left|\frac{x_{k}}{y_{k}}-L\right|\right) \geq \varepsilon\right\} \in \mathcal{I}
$$


(denoted by $\left.x_{k} \stackrel{\mathcal{I}\left(N_{\theta}^{f}\right)}{\sim} y_{k}\right)$ ) and simply strongly $f$-asymptotically lacunary $\mathcal{I}$-equivalent if $L=1$.

Two nonnegative double sequences $x=\left(x_{k j}\right)$ and $y=\left(y_{k j}\right)$ are said to be asymptotically strongly $\mathcal{I}_{2}$-equivalent of multiple $L$ if for every $\varepsilon>0$,

$$
\left\{(m, n) \in \mathbb{N} \times \mathbb{N}: \frac{1}{m n} \sum_{k, j=1}^{m, n}\left|\frac{x_{k j}}{y_{k j}}-L\right| \geq \varepsilon\right\} \in \mathcal{I}_{2}
$$

(denoted by $x_{k j} \stackrel{\left[\mathcal{I}^{L}\right]}{\sim} y_{k j}$ ) and simply asymptotically $\mathcal{I}_{2}$-statistical equivalent if $L=1$.

Two nonnegative double sequences $x=\left(x_{k j}\right)$ and $y=\left(y_{k j}\right)$ are said to be asymptotically $\mathcal{I}_{2}$-statistically equivalent of multiple $L$ if for all $\varepsilon>0$ and each $\gamma>0$,

$$
\left\{(m, n) \in \mathbb{N} \times \mathbb{N}: \frac{1}{m n}\left|\left\{k, j \leq m, n:\left|\frac{x_{k j}}{y_{k j}}-L\right| \geq \varepsilon\right\}\right| \geq \gamma\right\} \in \mathcal{I}_{2}
$$

(denoted by $x_{k j} \stackrel{\mathcal{I}_{2}(S)}{\sim} y_{k j}$ ) and simply asymptotically $\mathcal{I}_{2}$-statistically equivalent if $L=1$.

Two nonnegative double sequences $x=\left(x_{k j}\right)$ and $y=\left(y_{k j}\right)$ are said to be asymptotically lacunary $\mathcal{I}_{2}$-equivalent of multiple $L$ if for every $\varepsilon>0$,

$$
\left\{(r, u) \in \mathbb{N} \times \mathbb{N}: \frac{1}{h_{r u}} \sum_{(k, j) \in I_{r u}}\left|\frac{x_{k j}}{y_{k j}}-L\right| \geq \varepsilon\right\} \in \mathcal{I}_{2}
$$

(denoted by $x_{k j} \stackrel{\left[\mathcal{I}_{\theta_{2}}^{L}\right]}{\sim} y_{k j}$ ) and simply strongly asymptotically lacunary $\mathcal{I}_{2}$-equivalent if $L=1$.

Two nonnegative double sequences $x=\left(x_{k j}\right)$ and $y=\left(y_{k j}\right)$ are said to be asymptotically lacunary $\mathcal{I}_{2}$-statistically equivalent of multiple $L$ if for all $\varepsilon>0$ and $\gamma>0$,

$$
\left\{(r, u) \in \mathbb{N} \times \mathbb{N}: \frac{1}{h_{r u}}\left|\left\{(k, j) \in I_{r u}:\left|\frac{x_{k j}}{y_{k j}}-L\right| \geq \varepsilon\right\}\right| \geq \gamma\right\} \in \mathcal{I}_{2}
$$

(denoted by $x_{k j} \stackrel{\mathcal{I}_{2}\left(S_{\theta}\right)}{\sim} y_{k j}$ ) and simply asymptotically $\mathcal{I}_{2}$-statistically equivalent if $L=1$.

Lemma 1 ([37]) Let $f$ be a modulus, and let $0<\delta<1$. Then, for each $x \geq \delta$, we have $f(x) \leq$ $2 f(1) \delta^{-1} x$.

\section{Main results}

Definition 2.1 Let $f$ be a modulus function. Two nonnegative sequences $x=\left(x_{k j}\right)$ and $y=\left(y_{k j}\right)$ are said to be $f$-asymptotically $\mathcal{I}_{2}$-equivalent of multiple L if for every $\varepsilon>0$,

$$
\left\{(k, j) \in \mathbb{N} \times \mathbb{N}: f\left(\left|\frac{x_{k j}}{y_{k j}}-L\right|\right) \geq \varepsilon\right\} \in \mathcal{I}_{2}
$$

(denoted by $x_{k j} \stackrel{\mathcal{I}_{2}^{L}(f)}{\sim} y_{k j}$ ) and simply $f$-asymptotically $\mathcal{I}_{2}$-equivalent if $L=1$.

Definition 2.2 Let $f$ be a modulus function. The two nonnegative sequences $x=\left(x_{k j}\right)$ and $y=\left(y_{k j}\right)$ are said to be strongly $\mathrm{f}$-asymptotically $\mathcal{I}_{2}$-equivalent of multiple $L$ if for every 
$\varepsilon>0$,

$$
\left\{(m, n) \in \mathbb{N} \times \mathbb{N}: \frac{1}{m n} \sum_{k, j=1}^{m, n} f\left(\left|\frac{x_{k j}}{y_{k j}}-L\right|\right) \geq \varepsilon\right\} \in \mathcal{I}_{2}
$$

(denoted by $x_{k j} \stackrel{\left[\mathcal{I}_{2}^{L}(f)\right]}{\sim} y_{k j}$ ) and simply strongly $f$-asymptotically $\mathcal{I}_{2}$-equivalent if $L=1$.

Theorem 2.1 Letf be a modulus function. Then $x_{k j} \stackrel{\left[\mathcal{I}_{2}^{L}\right]}{\sim} y_{k j} \Rightarrow x_{k j} \stackrel{\left[\mathcal{I}_{2}^{L}(f)\right]}{\sim} y_{k j}$.

Proof Suppose that $x_{k j} \stackrel{\left[\mathcal{I}_{2}^{L}\right]}{\sim} y_{k j}$, and let $\varepsilon>0$ be given. Select $0<\delta<1$ such that $f(t)<\varepsilon$ for $0 \leq t \leq \delta$. We can write

$$
\begin{aligned}
\frac{1}{m n} \sum_{k, j=1}^{m, n} f\left(\left|\frac{x_{k j}}{y_{k j}}-L\right|\right)= & \frac{1}{m n} \sum_{\substack{k, j=1 \\
\mid x_{k j} \\
y_{k j}}-L \mid \leq \delta}^{m, n} f\left(\left|\frac{x_{k j}}{y_{k j}}-L\right|\right) \\
& +\frac{1}{m n} \sum_{\substack{k, j=1 \\
\left|\frac{x_{k j}}{y_{k j}}-L\right|>\delta}}^{m, n} f\left(\left|\frac{x_{k j}}{y_{k j}}-L\right|\right),
\end{aligned}
$$

and so by Lemma 1

$$
\frac{1}{m n} \sum_{k, j=1}^{m, n} f\left(\left|\frac{x_{k j}}{y_{k j}}-L\right|\right)<\varepsilon+\left(\frac{2 f(1)}{\delta}\right) \frac{1}{m n} \sum_{k, j=1}^{m, n}\left|\frac{x_{k j}}{y_{k j}}-L\right| .
$$

Thus, for any $\gamma>0$,

$$
\begin{aligned}
& \left\{(m, n) \in \mathbb{N} \times \mathbb{N}: \frac{1}{m n} \sum_{k, j=1}^{m, n} f\left(\left|\frac{x_{k j}}{y_{k j}}-L\right|\right) \geq \gamma\right\} \\
& \subseteq\left\{(m, n) \in \mathbb{N} \times \mathbb{N}: \frac{1}{m n} \sum_{k, j=1}^{m, n}\left|\frac{x_{k j}}{y_{k j}}-L\right| \geq \frac{(\gamma-\varepsilon) \delta}{2 f(1)}\right\} .
\end{aligned}
$$

Since $x_{k j} \stackrel{\left[\mathcal{I}_{2}^{L}\right]}{\sim} y_{k j}$, it follows that the second set and thus the first set in the above expression belong to $\mathcal{I}_{2}$. This proves that $x_{k j} \stackrel{\left[\mathcal{I}_{2}^{L}(f)\right]}{\sim} y_{k j}$.

Theorem 2.2 If $\lim _{t \rightarrow \infty} \frac{f(t)}{t}=\alpha>0$, then $x_{k j} \stackrel{\left[\mathcal{I}_{2}^{L}(f)\right]}{\sim} y_{k j} \Leftrightarrow x_{k j} \stackrel{\left[\mathcal{I}_{2}^{L}\right]}{\sim} y_{k j}$.

Proof We showed that $x_{k j} \stackrel{\left[\mathcal{I}_{2}^{L}\right]}{\sim} y_{k j} \Rightarrow x_{k j} \stackrel{\left[\mathcal{I}_{2}^{L}(f)\right]}{\sim} y_{k j}$ in Theorem 2.1. Now we must show that $x_{k j} \stackrel{\left[\mathcal{I}_{2}^{L}(f)\right]}{\sim} y_{k j} \Rightarrow x_{k j} \stackrel{\left[\mathcal{I}_{2}^{L}\right]}{\sim} y_{k j}$.

Let $\lim _{t \rightarrow \infty} \frac{f(t)}{t}=\alpha>0$. Then we have $f(t) \geq \alpha t$ for all $t \geq 0$. Assume that $x_{k j} \stackrel{\left[\mathcal{I}_{2}^{L}(f)\right]}{\sim} y_{k j}$. Since

$$
\frac{1}{m n} \sum_{k, j=1}^{m, n} f\left(\left|\frac{x_{k j}}{y_{k j}}-L\right|\right) \geq \frac{1}{m n} \sum_{k, j=1}^{m, n} \alpha\left(\left|\frac{x_{k j}}{y_{k j}}-L\right|\right)=\alpha\left(\frac{1}{m n} \sum_{k, j=1}^{m, n}\left|\frac{x_{k j}}{y_{k j}}-L\right|\right),
$$


it follows that for each $\varepsilon>0$, we have

$$
\begin{aligned}
& \left\{(m, n) \in \mathbb{N} \times \mathbb{N}: \frac{1}{m n} \sum_{k, j=1}^{m, n}\left|\frac{x_{k j}}{y_{k j}}-L\right| \geq \varepsilon\right\} \\
& \subseteq\left\{(m, n) \in \mathbb{N} \times \mathbb{N}: \frac{1}{m n} \sum_{k, j=1}^{m, n} f\left(\left|\frac{x_{k j}}{y_{k j}}-L\right|\right) \geq \alpha \varepsilon\right\} .
\end{aligned}
$$

Since $x_{k j} \stackrel{\left[\mathcal{I}_{2}^{L}(f)\right]}{\sim} y_{k j}$, it follows that the latter set and hence the former set in the above expression belong to $\mathcal{I}_{2}$. This proves that $x_{k j} \stackrel{\left[\mathcal{I}_{2}^{L}(f)\right]}{\sim} y_{k j} \Leftrightarrow x_{k j}{\stackrel{\left[\mathcal{I}_{2}^{L}\right]}{\sim}}_{y_{k j}}$.

Definition 2.3 Let $f$ be a modulus function. Two nonnegative sequences $x=\left(x_{k j}\right)$ and $y=\left(y_{k j}\right)$ are said to be $f$-asymptotically lacunary $\mathcal{I}_{2}$-equivalent of multiple $L$ if for every $\varepsilon>0$,

$$
\left\{(k, j) \in I_{r u}: f\left(\left|\frac{x_{k j}}{y_{k j}}-L\right|\right) \geq \varepsilon\right\} \in \mathcal{I}_{2}
$$

(denoted by $\left.x_{k j} \stackrel{\mathcal{I}_{\theta_{2}}^{L}(f)}{\sim} y_{k j}\right)$ ) and simply $f$-asymptotically lacunary $\mathcal{I}_{2}$-equivalent if $L=1$.

Definition 2.4 Let $f$ be a modulus function. Two nonnegative sequences $x=\left(x_{k j}\right)$ and $y=\left(y_{k j}\right)$ are said to be strongly $f$-asymptotically lacunary $\mathcal{I}_{2}$-equivalent of multiple $L$ if for every $\varepsilon>0$,

$$
\left\{(r, u) \in \mathbb{N} \times \mathbb{N}: \frac{1}{h_{r u}} \sum_{(k, j) \in I_{r u}} f\left(\left|\frac{x_{k j}}{y_{k j}}-L\right|\right) \geq \varepsilon\right\} \in \mathcal{I}_{2}
$$

(denoted by $\left.x_{k j} \stackrel{\left[\mathcal{I}_{\theta_{2}}^{L}(f)\right]}{\sim} y_{k j}\right)$ ) and simply strongly $f$-asymptotically lacunary $\mathcal{I}_{2}$-equivalent if $L=1$.

Theorem 2.3 Letf be a modulus function. Then, $x_{k j} \stackrel{\left[\mathcal{I}_{\theta_{2}}^{L}\right]}{\sim} y_{k j} \Rightarrow x_{k j} \stackrel{\left[\mathcal{I}_{\theta_{2}}^{L}(f)\right]}{\sim} y_{k j}$.

Proof Let $x_{k j} \stackrel{\left[\mathcal{I}_{\theta_{2}}^{L}\right]}{\sim} y_{k j}$, and let $\varepsilon>0$ be given. Choose $0<\delta<1$ such that $f(t)<\varepsilon$ for $0 \leq t \leq \delta$. We can write

$$
\begin{aligned}
\frac{1}{h_{r u}} \sum_{(k, j) \in I_{r u}} f\left(\left|\frac{x_{k j}}{y_{k j}}-L\right|\right)= & \frac{1}{h_{r u}} \sum_{\substack{(k, j) \in I_{r u} \\
\left|\frac{x_{k j}}{y_{k j}}-L\right| \leq \delta}} f\left(\left|\frac{x_{k j}}{y_{k j}}-L\right|\right) \\
& +\frac{1}{h_{r u}} \sum_{\substack{(k, j) \in I_{r u} \\
\left|\frac{x_{k j}}{y_{k j}}-L\right|>\delta}} f\left(\left|\frac{x_{k j}}{y_{k j}}-L\right|\right),
\end{aligned}
$$

and so by Lemma 1

$$
\frac{1}{h_{r u}} \sum_{(k, j) \in I_{r u}} f\left(\left|\frac{x_{k j}}{y_{k j}}-L\right|\right)<\varepsilon+\left(\frac{2 f(1)}{\delta}\right) \frac{1}{h_{r u}} \sum_{(k, j) \in I_{r}}\left|\frac{x_{k j}}{y_{k j}}-L\right| .
$$


Thus, for each any $\gamma>0$,

$$
\begin{aligned}
& \left\{(r, u) \in \mathbb{N} \times \mathbb{N}: \frac{1}{h_{r u}} \sum_{(k, j) \in I_{r u}} f\left(\left|\frac{x_{k j}}{y_{k j}}-L\right|\right) \geq \gamma\right\} \\
& \subseteq\left\{(r, u) \in \mathbb{N} \times \mathbb{N}: \frac{1}{h_{r u}} \sum_{(k, j) \in I_{r u}}\left|\frac{x_{k j}}{y_{k j}}-L\right| \geq \frac{(\gamma-\varepsilon) \delta}{2 f(1)}\right\} .
\end{aligned}
$$

Since $x_{k j} \stackrel{\left[\mathcal{I}_{\theta_{2}}^{L}\right]}{\sim} y_{k j}$, it follows that the latter set and hence the former set in the above expression belong to $\mathcal{I}_{2}$. This proves that $x_{k j} \stackrel{\left[\mathcal{I}_{\theta_{2}}^{L}(f)\right]}{\sim} y_{k j}$.

Theorem 2.4 If $\lim _{t \rightarrow \infty} \frac{f(t)}{t}=\alpha>0$, then $x_{k j} \stackrel{\left[\mathcal{I}_{\theta_{2}}^{L}(f)\right]}{\sim} y_{k j} \Leftrightarrow x_{k j} \stackrel{\left[\mathcal{I}_{\theta_{2}}^{L}\right]}{\sim} y_{k j}$.

Proof We showed that $x_{k j} \stackrel{\left[\mathcal{I}_{\theta_{2}}^{L}\right]}{\sim} y_{k j} \Rightarrow x_{k j} \stackrel{\left[\mathcal{I}_{\theta_{2}}^{L}(f)\right]}{\sim} y_{k j}$ in Theorem 2.3. Now we must show that $x_{k j} \stackrel{\left[\mathcal{I}_{\theta_{2}}^{L}(f)\right]}{\sim} y_{k j} \Rightarrow x_{k j} \stackrel{\left[\mathcal{I}_{\theta_{2}}^{L}\right]}{\sim} y_{k j}$.

Let $\lim _{t \rightarrow \infty} \frac{f(t)}{t}=\alpha>0$. Then we have $f(t) \geq \alpha t$ for all $t \geq 0$. Assume that $x_{k j} \stackrel{\left[\mathcal{I}_{\theta_{2}}^{L}(f)\right]}{\sim} y_{k j}$. From

$$
\begin{aligned}
\frac{1}{h_{r u}} \sum_{(k, j) \in I_{r u}} f\left(\left|\frac{x_{k j}}{y_{k j}}-L\right|\right) & \geq \frac{1}{h_{r u}} \sum_{(k, j) \in I_{r u}} \alpha\left(\left|\frac{x_{k j}}{y_{k j}}-L\right|\right) \\
& =\alpha\left(\frac{1}{h_{r u}} \sum_{(k, j) \in I_{r u}}\left|\frac{x_{k j}}{y_{k j}}-L\right|\right)
\end{aligned}
$$

it follows that for each $\varepsilon>0$, we have

$$
\begin{aligned}
& \left\{(r, u) \in \mathbb{N} \times \mathbb{N}: \frac{1}{h_{r u}} \sum_{(k, j) \in I_{r u}}\left|\frac{x_{k j}}{y_{k j}}-L\right| \geq \varepsilon\right\} \\
& \quad \subseteq\left\{(r, u) \in \mathbb{N} \times \mathbb{N}: \frac{1}{h_{r u}} \sum_{(k, j) \in I_{r u}} f\left(\left|\frac{x_{k j}}{y_{k j}}-L\right|\right) \geq \alpha \varepsilon\right\} .
\end{aligned}
$$

Since $x_{k j} \stackrel{\left[\mathcal{I}_{\theta_{2}}^{L}(f)\right]}{\sim} y_{k j}$, it follows that the latter set and hence the former set in the above expression belong to $\mathcal{I}_{2}$. This proves that $x_{k j} \stackrel{\left[\mathcal{I}_{\theta_{2}}^{L}\right]}{\sim} y_{k j} \Leftrightarrow x_{k j} \stackrel{\left[\mathcal{I}_{\theta_{2}}^{L}(f)\right]}{\sim} y_{k j}$.

Theorem 2.5 Letf be a modulus function. If $\liminf _{r, u} q_{r, u}>1$, then

$$
x_{k j} \stackrel{\left[\mathcal{I}_{2}^{L}(f)\right]}{\sim} y_{k j} \Rightarrow x_{k j} \stackrel{\left[\mathcal{I}_{\theta_{2}}^{L}(f)\right]}{\sim} y_{k j}
$$

Proof Suppose that $\liminf _{r, u} q_{r, u}>1$. Then there exists $\eta>0$ such that $q_{r, u} \geq 1+\eta$ for sufficiently large $r, u$. Then we have

$$
\frac{h_{r u}}{k_{r} j_{u}} \geq \frac{\eta}{1+\eta}
$$


Let $x_{k j} \stackrel{\left[\mathcal{I}_{2}^{L}(f)\right]}{\sim} y_{k j}$. For sufficiently large $r, u$, we have

$$
\begin{aligned}
\frac{1}{k_{r} j_{u}} \sum_{k, j=1,1}^{k_{r} j_{u}} f\left(\left|\frac{x_{k j}}{y_{k j}}-L\right|\right) & \geq \frac{1}{k_{r} j_{u}} \sum_{(k, j) \in I_{r u}} f\left(\left|\frac{x_{k j}}{y_{k j}}-L\right|\right) \\
& =\left(\frac{h_{r u}}{k_{r} j_{u}}\right) \frac{1}{h_{r u}} \sum_{(k, j) \in I_{r u}} f\left(\left|\frac{x_{k j}}{y_{k j}}-L\right|\right) \\
& \geq \frac{\eta}{1+\eta} \frac{1}{h_{r u}} \sum_{(k, j) \in I_{r u}} f\left(\left|\frac{x_{k j}}{y_{k j}}-L\right|\right),
\end{aligned}
$$

which gives, for any $\varepsilon>0$,

$$
\begin{aligned}
& \left\{(r, u) \in \mathbb{N} \times \mathbb{N}: \frac{1}{h_{r u}} \sum_{(k, j) \in I_{r u}} f\left(\left|\frac{x_{k j}}{y_{k j}}-L\right|\right) \geq \varepsilon\right\} \\
& \quad \subseteq\left\{(r, u) \in \mathbb{N} \times \mathbb{N}: \frac{1}{k_{r} j_{u}} \sum_{k, j=1,1}^{k_{r} j_{u}} f\left(\left|\frac{x_{k j}}{y_{k j}}-L\right|\right) \geq \frac{\varepsilon \eta}{1+\eta}\right\} .
\end{aligned}
$$

Since $x_{k j} \stackrel{\left[\mathcal{I}_{2}^{L}(f)\right]}{\sim} y_{k j}$, it follows that the latter set and hence the former set belong to $\mathcal{I}_{2}$. This shows that $x_{k j} \stackrel{\left[\mathcal{I}_{\theta_{2}}^{L}(f)\right]}{\sim} y_{k j}$.

Theorem 2.6 Letf be a modulus function. Then, $x_{k j} \stackrel{\left[\mathcal{I}_{2}^{L}(f)\right]}{\sim} y_{k j} \Rightarrow x_{k j} \stackrel{\mathcal{I}_{2}(S)}{\sim} y_{k j}$.

Proof Assume that $x_{k j} \stackrel{\left[\mathcal{I}_{2}^{L}(f)\right]}{\sim} y_{k j}$, and let $\varepsilon>0$ be given. From

$$
\begin{aligned}
\frac{1}{m n} \sum_{k, j=1}^{m, n} f\left(\left|\frac{x_{k j}}{y_{k j}}-L\right|\right) & \geq \frac{1}{m n} \sum_{\substack{k, j=1 \\
\left|\frac{x_{k j}}{y_{k j}}-L\right| \geq \varepsilon}}^{n} f\left(\left|\frac{x_{k j}}{y_{k j}}-L\right|\right) \\
& \geq f(\varepsilon) \cdot \frac{1}{m n}\left|\left\{k \leq m, j \leq n:\left|\frac{x_{k j}}{y_{k j}}-L\right| \geq \varepsilon\right\}\right|
\end{aligned}
$$

it follows that for any $\gamma>0$, we have

$$
\begin{aligned}
& \left\{(m, n) \in \mathbb{N} \times \mathbb{N}: \frac{1}{m n}\left|\left\{k \leq m, j \leq n:\left|\frac{x_{k j}}{y_{k j}}-L\right| \geq \varepsilon\right\}\right| \geq \frac{\gamma}{f(\varepsilon)}\right\} \\
& \subseteq\left\{(m, n) \in \mathbb{N} \times \mathbb{N}: \frac{1}{m n} \sum_{k, j=1}^{m, n} f\left(\left|\frac{x_{k j}}{y_{k j}}-L\right|\right) \geq \gamma\right\} .
\end{aligned}
$$

Since $x_{k j} \stackrel{\left[\mathcal{I}_{2}^{L}(f)\right]}{\sim} y_{k j}$, it follows the latter set and hence the former set in the above expression belong to $\mathcal{I}_{2}$. Therefore $x_{k j} \stackrel{\mathcal{I}_{2}(S)}{\sim} y_{k j}$.

Theorem 2.7 Let $f$ be a modulus function. Iff is bounded, then $x_{k j} \stackrel{\mathcal{I}_{2}(S)}{\sim} y_{k j} \Leftrightarrow x_{k j} \stackrel{\left[\mathcal{I}_{2}^{L}(f)\right]}{\sim} y_{k j}$. 
Proof We showed that $x_{k j} \stackrel{\left[\mathcal{I}_{2}^{L}(f)\right]}{\sim} y_{k j} \Rightarrow x_{k j} \stackrel{\mathcal{I}_{2}(S)}{\sim} y_{k j}$ in Theorem 2.6. Now we must show that $x_{k j} \stackrel{\mathcal{I}_{2}(S)}{\sim} y_{k j} \Rightarrow x_{k j} \stackrel{\left[\mathcal{I}_{2}^{L}(f)\right]}{\sim} y_{k j}$

Assume that $f$ is bounded and let $x_{k j} \stackrel{\mathcal{I}_{2}(S)}{\sim} y_{k j}$. Since $f$ is bounded, there exists a positive real number $M$ such that $|f(x)| \leq M$ for all $x \geq 0$. We have

$$
\begin{aligned}
\frac{1}{m n} \sum_{k, j=1}^{m, n} f\left(\left|\frac{x_{k j}}{y_{k j}}-L\right|\right)= & \frac{1}{m n} \sum_{\substack{k, j=1 \\
\left|\frac{x_{k j}}{y_{k j}}-L\right| \geq \varepsilon}}^{m, n} f\left(\left|\frac{x_{k j}}{y_{k j}}-L\right|\right) \\
& +\frac{1}{m n} \sum_{\substack{k, j=1 \\
x_{k j}}}^{m, n} f\left(\left|\frac{x_{k j}}{y_{k j}}-L\right|\right) \\
& \leq \frac{M}{m n}\left|\left\{k \leq m, j \leq n:\left|\frac{x_{k j}}{y_{k j}}-L\right| \geq \varepsilon\right\}\right|+f(\varepsilon) .
\end{aligned}
$$

This proves that $x_{k j} \stackrel{\left[\mathcal{I}_{2}^{L}(f)\right]}{\sim} y_{k j}$.

Theorem 2.8 Letf be a modulus function. Then $x_{k j} \stackrel{\left[\mathcal{I}_{\theta_{2}}^{L}(f)\right]}{\sim} y_{k j} \Rightarrow x_{k j} \stackrel{\mathcal{I}\left(S_{\theta_{2}}\right)}{\sim} y_{k j}$.

Proof Assume that $x_{k} \stackrel{\left[\mathcal{I}_{\theta_{2}}^{L}(f)\right]}{\sim} y_{k}$, and let $\varepsilon>0$ be given. From

$$
\begin{aligned}
\frac{1}{h_{r u}} \sum_{(k, j) \in I_{r u}} f\left(\left|\frac{x_{k j}}{y_{k j}}-L\right|\right) & \geq \frac{1}{h_{r u}} \sum_{\substack{(k, j) \in I_{r u} \\
\left|\frac{x_{k j}}{y_{k j}}-L\right| \geq \varepsilon}} f\left(\left|\frac{x_{k j}}{y_{k j}}-L\right|\right) \\
& \geq f(\varepsilon) \cdot \frac{1}{h_{r u}}\left|\left\{(k, j) \in I_{r u}:\left|\frac{x_{k j}}{y_{k j}}-L\right| \geq \varepsilon\right\}\right|
\end{aligned}
$$

it follows that for any $\gamma>0$,

$$
\begin{aligned}
& \left\{(r, u) \in \mathbb{N} \times \mathbb{N}: \frac{1}{h_{r u}}\left|\left\{(k, j) \in I_{r u}:\left|\frac{x_{k j}}{y_{k j}}-L\right| \geq \varepsilon\right\}\right| \geq \gamma\right\} \\
& \subseteq\left\{(r, u) \in \mathbb{N} \times \mathbb{N}: \frac{1}{h_{r u}} \sum_{(k, j) \in I_{r u}} f\left(\left|\frac{x_{k j}}{y_{k j}}-L\right|\right) \geq \gamma f(\varepsilon)\right\} .
\end{aligned}
$$

Since $x_{k j} \stackrel{\left[\mathcal{I}_{\theta_{2}}^{L}(f)\right]}{\sim} y_{k j}$, the last set belongs to $\mathcal{I}_{2}$, and so by the definition of an ideal the first set belongs to $\mathcal{I}_{2}$. Therefore $x_{k j} \stackrel{\mathcal{I}\left(S_{\theta_{2}}\right)}{\sim} y_{k j}$.

Theorem 2.9 Letf be a modulus function. Iff is bounded, then $x_{k j} \stackrel{\mathcal{I}\left(S_{\theta_{2}}\right)}{\sim} y_{k j} \Leftrightarrow x_{k j} \stackrel{\left[\mathcal{I}_{\theta_{2}}^{L}(f)\right]}{\sim} y_{k j}$. Proof We showed that $x_{k j} \stackrel{\left[\mathcal{I}_{\theta_{2}}^{L}(f)\right]}{\sim} y_{k j} \Rightarrow x_{k j} \stackrel{\mathcal{I}\left(S_{\theta_{2}}\right)}{\sim} y_{k j}$ in Theorem 2.8. Now we must show that $x_{k j} \stackrel{\mathcal{I}\left(S_{\theta_{2}}\right)}{\sim} y_{k j} \Rightarrow x_{k j} \stackrel{\left[\mathcal{I}_{\theta_{2}}^{L}(f)\right]}{\sim} y_{k j}$. 
Assume that $f$ is bounded and let $x_{k j} \stackrel{\mathcal{I}\left(S_{\theta_{2}}\right)}{\sim} y_{k j}$. Since $f$ is bounded, there exists a positive real number $M$ such that $|f(x)| \leq M$ for all $x \geq 0$. We have

$$
\begin{aligned}
& \frac{1}{h_{r u}} \sum_{(k, j) \in I_{r u}} f\left(\left|\frac{x_{k j}}{y_{k j}}-L\right|\right)=\frac{1}{h_{r u}} \sum_{(k, j) \in I_{r u}} f\left(\left|\frac{x_{k j}}{y_{k j}}-L\right|\right) \\
& \left|\frac{x_{k j}}{y_{k j}}-L\right| \geq \varepsilon \\
& +\frac{1}{h_{r u}} \sum_{(k, j) \in I_{r u}} f\left(\left|\frac{x_{k j}}{y_{k j}}-L\right|\right) \\
& \left|\frac{x_{k j}}{y_{k j}}-L\right|<\varepsilon \\
& \leq \frac{M}{h_{r u}}\left|\left\{(k, j) \in I_{r u}:\left|\frac{x_{k j}}{y_{k j}}-L\right| \geq \varepsilon\right\}\right|+f(\varepsilon) .
\end{aligned}
$$

This proves that $x_{k j} \stackrel{\left[\mathcal{I}_{\theta_{2}}^{L}(f)\right]}{\sim} y_{k j}$.

\section{Acknowledgements}

I would like to express my thanks to Professor Erdinç Dündar, Department of Mathematics, Afyon Kocatepe University, Afyonkarahisar, Turkey, for his careful reading of an earlier version of this paper and constructive comments, which improved the presentation of the paper. Also, I highly appreciate anonymous reviewers for helpful comments and valuable suggestions.

\section{Funding}

This work is not sponsored by any institution.

\section{Availability of data and materials}

Not applicable.

\section{Competing interests}

The author declares to have no competing interests.

\section{Authors' contributions}

The author was the only one to contribute to the writing of this paper. The author read and approved the final manuscript.

\section{Publisher's Note}

Springer Nature remains neutral with regard to jurisdictional claims in published maps and institutional affiliations.

Received: 20 May 2019 Accepted: 7 August 2019 Published online: 22 August 2019

\section{References}

1. Belen, C., Mohiuddine, S.A.: Generalized weighted statistical convergence and application. Appl. Math. Comput. 219(18), 9821-9826 (2013)

2. Braha, N.L., Srivastava, H.M., Mohiuddine, S.A.: A Korovkin's type approximation theorem for periodic functions via the statistical summability of the generalized De La Vallée Poussin mean. Appl. Math. Comput. 228, 162-169 (2014)

3. Connor, J.S.: On strong matrix summability with respect to a modulus and statistical convergence. Can. Math. Bull. 32 194-198 (1989)

4. Das, P., Kostyrko, P., Wilczyński, W., Malik, P.: / and /*-convergence of double sequences. Math. Slovaca 58(5), 605-620 (2008)

5. Dündar, E.: On rough $\mathcal{I}_{2}$-convergence of double sequences. Numer. Funct. Anal. Optim. 37(4), 480-491 (2016)

6. Dündar, E., Altay, B.: On some properties of $\mathcal{I}_{2}$-convergence and $\mathcal{I}_{2}$-Cauchy of double sequences. Gen. Math. Notes 7(1), 1-12 (2011)

7. Dündar, E., Altay, B.: Multipliers for bounded $\mathcal{I}_{2}$-convergent of double sequences. Math. Comput. Model. 55(3-4), 1193-1198 (2012)

8. Dündar, E., Altay, B.: $\mathcal{I}_{2}$-Convergence and $\mathcal{I}_{2}$-Cauchy of double sequences. Acta Math. Sci. 34B(2), $343-353$ (2014)

9. Dündar, E., Altay, B.: $\mathcal{I}_{2}$-Convergence of double sequences of functions. Electron. J. Math. Anal. Appl. 3(1), 111-121 (2015)

10. Dündar, E., Altay, B.: $\mathcal{I}_{2}$-Uniform convergence of double sequences of functions. Filomat 30(5), 1273-1281 (2016)

11. Dündar, E., Talo, Ö.: $\mathcal{I}_{2}$-Convergence of double sequences of fuzzy numbers. Iran. J. Fuzzy Syst. 10(3), 37-50 (2013)

12. Dündar, E., Ulusu, U., Nuray, F.: On asymptotically ideal invariant equivalence of double sequences. (Under review)

13. Edely, O.H.H., Mohiuddine, S.A., Noman, A.K.: Korovkin type approximation theorems obtained through generalized statistical convergence. Appl. Math. Lett. 23, 1382-1387 (2010) 
14. Fast, H.: Sur la convergence statistique. Colloq. Math. 2, 241-244 (1951)

15. Fridy, J.A., Orhan, C.: Lacunary statistical convergence. Pac. J. Math. 160, 43-51 (1993)

16. Hazarika, B., Kumar, V.: On asymptotically double lacunary statistical equivalent sequences in ideal context. J. Inequal. Appl. 2013, 543 (2013). https://doi.org/10.1186/1029-242X-2013-543

17. Khan, V.A., Khan, N.: On some $\mathcal{I}$-convergent double sequence spaces defined by a modulus function. Engineering 5 , 35-40 (2013)

18. Kılınç, G., Solak, I.: Some double sequence spaces defined by a modulus function. Gen. Math. Notes 25(2), 19-30 (2014)

19. Kolk, E.: On strong boundedness and summability with respect to a sequence of moduli. Tartu UI. Toimetised. 960 , 41-50 (1993)

20. Kostyrko, P., Šalát, T., Wilczyński, W.: I-Convergence. Real Anal. Exch. 26(2), 669-686 (2000)

21. Kumar, V., Sharma, A.: Asymptotically lacunary equivalent sequences defined by ideals and modulus function. Math. Sci. 6, 23 (2012)

22. Lorentz, G.: A contribution to the theory of divergent sequences. Acta Math. 80, 167-190 (1948)

23. Maddox, I.J.: Sequence spaces defined by a modulus. Math. Proc. Camb. Philos. Soc. 100, 161-166 (1986)

24. Marouf, M.: Asymptotic equivalence and summability. Int. J. Math. Math. Sci. 16(4), 755-762 (1993)

25. Mohiuddine, S.A., Alamri, B.A.S.: Generalization of equi-statistical convergence via weighted lacunary sequence with associated Korovkin and Voronovskaya type approximation theorems. Rev. R. Acad. Cienc. Exactas Fís. Nat., Ser. A Mat. $113,1955-1973(2019)$

26. Mohiuddine, S.A., Alotaibi, A., Mursaleen, M.: Statistical convergence of double sequences in locally solid Riesz spaces. Abstr. Appl. Anal. 2012, Article ID 719729 (2012)

27. Mohiuddine, S.A., Asiri, A., Hazarika, B.: Weighted statistical convergence through difference operator of sequences of fuzzy numbers with application to fuzzy approximation theorems. Int. J. Gen. Syst. 48(5), 492-506 (2019)

28. Mursaleen, M., Edely, O.H.H.: Statistical convergence of double sequences. J. Math. Anal. Appl. 288, $223-231$ (2003)

29. Mursaleen, M., Mohiuddine, S.A.: Statistical convergence of double sequences in intuitionistic fuzzy normed spaces. Chaos Solitons Fractals 41, 2414-2421 (2009)

30. Mursaleen, M., Mohiuddine, S.A.: On lacunary statistical convergence with respect to the intuitionistic fuzzy normed space. J. Comput. Appl. Math. 233, 142-149 (2009)

31. Mursaleen, M., Mohiuddine, S.A.: On ideal convergence of double sequences in probabilistic normed spaces. Mathem. Rep. 12(62) 4, 359-371 (2010)

32. Mursaleen, M., Mohiuddine, S.A.: On ideal convergence in probabilistic normed spaces. Math. Slovaca 62(1), 49-62 (2012)

33. Nakano, H.: Concave modulars. J. Math. Soc. Jpn. 5, 29-49 (1953)

34. Nuray, F., Ulusu, U., Dündar, E.: Lacunary statistical convergence of double sequences of sets. Soft Comput. 20(7), 2883-2888 (2016). https://doi.org/10.1007/s00500-015-1691-8

35. Patterson, R.F.: On asymptotically statistically equivalent sequences. Demostratio Mathematica 36(1), 149-153 (2003)

36. Patterson, R.F., Savaş, E.: On asymptotically lacunary statistically equivalent sequences. Thai J. Math. 4(2), 267-272 (2006)

37. Pehlivan, S., Fisher, B.: Some sequences spaces defined by a modulus. Math. Slovaca 45, 275-280 (1995)

38. Savaş, E.: On $\mathcal{I}$-asymptotically lacunary statistical equivalent sequences. Adv. Differ. Equ. 2013, 111 (2013). https://doi.org/10.1186/1687-1847-2013-111

39. Schoenberg, I.J.: The integrability of certain functions and related summability methods. Am. Math. Mon. 66, 361-375 (1959)

40. Tortop, S.., Dündar, E.: Wijsman $\mathcal{I}_{2}$-invariant convergence of double sequences of sets. J. Inequal. Spec. Funct. 9(4), 90-100 (2018)

41. Türkmen, M.R., Dündar, E.: On lacunary statistical convergence of double sequences and some properties in fuzzy normed spaces. J. Intell. Fuzzy Syst. 36(2), 1683-1690 (2019)

42. Ulusu, U., Dündar, E.: Asymptotically lacunary $\mathcal{I}_{2}$-invariant equivalence. J. Intell. Fuzzy Syst. 36(1), 467-472 (2019)

43. Ulusu, U., Dündar, E., Nuray, F.: Lacunary $\mathcal{I}_{2}$-invariant convergence and some properties. Int. J. Anal. Appl. 16(3), 317-327 (2018)

44. Yegül, S., Dündar, E.: Statistical convergence of double sequences of functions and some properties in 2-normed spaces. Facta Univ., Ser. Math. Inform. 33(5), 705-719 (2018)

\section{Submit your manuscript to a SpringerOpen ${ }^{\circ}$ journal and benefit from:}

- Convenient online submission

- Rigorous peer review

- Open access: articles freely available online

- High visibility within the field

- Retaining the copyright to your article

Submit your next manuscript at $\boldsymbol{s p r i n g e r o p e n . c o m ~}$ 